\title{
"Crossed homonymous hemianopia" and "crossed left hemispatial neglect" in a case of Marchiafava-Bignami disease
}

\author{
Mutsuo Kamaki, Mitsuru Kawamura, Hirobumi Moriya, Keizo Hirayama
}

\begin{abstract}
"Crossed homonymous hemianopia" and "crossed left hemispatial neglect" were observed in a woman with Marchiafava-Bignami disease. Two forms of "crossed homonymous hemianopia" were observed. Initially, Goldmann perimeter testing showed a left homonymous hemianopia with the right hand and vice versa. Later, confrontation tests showed a left homonymous hemianopia, whereas visual field testing using the Goldmann perimeter (kinetic quantitative perimeter) and the OCTOPUS (Interzeag AG, static automated perimeter) showed a right homonymous hemianopia with either hand. "Crossed left hemispatial neglect" was not seen with the left hand, but neglect of the left hemifield was seen with the right hand. CT and MRI showed a lesion occupying almost the entire corpus callosum. PET showed no significant differences between comparable areas of the left and right cerebral hemispheres. These findings indicate that both signs of interhemispheric disconnection were due to the callosal lesion. Moreover, the "crossed left hemispatial neglect" can be explained as being a consequence of the dominance of the right cerebral hemisphere for visuospatial recognition.
\end{abstract}

(F Neurol Neurosurg Psychiatry 1993;56:1027-1032)

Department of

Neuropsychiatry,

Tokyo Metropolitan

Hiroo General

Hospital, Tokyo, Japan

M Kamaki

H Moriya

Department of

Neurology, School of

Medicine, Chiba

University, Chiba,

Japan

M Kawamura

K Hirayama

Correspondence to:

Dr Kamaki, Department of

Neuropsychiatry, Tokyo

Metropolitan Hiroo General

Hospital, 2-34-10 Ebisu,

Shibuya-ku, Tokyo 150,

Japan

Received 20 May 1992 and in revised form

21 September 1992

Accepted 11 December 1992

"Crossed homonymous hemianopia" and "crossed left hemispatial neglect" were observed in a case of Marchiafava-Bignami disease (MBD). MBD is a disease of chronic alcoholics caused by demyelination of the corpus callosum leading to symmetrical necrosis. The subject was a 43 year old woman with a history of alcohol abuse in which the callosal lesion was clearly diagnosed by $X$ ray CT and MRI. Both Goldmann and OCTOPUS perimetry were used according to their specified conditions. Use of the non-dominant hand was a modification made for this study. Initially, Goldmann perimetry showed a left homonymous hemianopia with the right hand pushing the switch and a right homonymous hemianopia with the left hand pushing the switch. Subsequently, visual field testing using instruments such as the Goldmann perimeter and the OCTOPUS static automated perimeter showed a right homonymous hemianopia with either hand pushing the switch. "Crossed left hemispatial neglect" was seen with the right hand when copying figures and during Albert's line-crossing test. Neither finding has previously been reported.

\section{Case report}

A 43 year old right handed housewife was found unconscious at home on 19 November 1987. She did not respond to her name and had been incontinent. She was taken to Tokyo Metropolitan Hiroo General Hospital. The patient had been a heavy drinker of Japanese rice wine for 20 years. She had complained of coldness and pain in the tips of her toes for three years, and had been aware of increasing forgetfulness over the preceding two months.

On admission, she was delirious, her speech was abnormal and she was unable to obey verbal commands. There were no abnormalities of the ocular fundi, the pupils were round and isocoric, and the light reflexes were normal. Central type left facial paresis and mildly decreased muscle strength of the left upper and lower limbs were found. General muscle tone was mildly hypertonic. Deep tendon reflexes were brisk, but both plantar responses were flexor. The sensory system could not be assessed and there was urinary incontinence.

One week after onset, her level of contemporal and spatial orientation remained. Her memory was still defective and her speech was dysarthric although improved. On confrontation tests a left homonymous hemianopia was found. The muscle strength of the left side of the face and left upper and lower limbs had improved, and muscle tone had returned to normal. Subsequently extinction of tactile stimuli of the left hand was found. There were digital disturbances of temperature and pain in all four limbs indicating a polyneuropathy. She regained control of her bladder and bowels. One month after onset, her level of consciousness was normal and she was cooperative enought to participate in neuropsychological testing. 
Neuroradiological finding

One month after onset, sagittal T1-weighted MRI $(T R=2080 \mathrm{~ms}$, TI = $500 \mathrm{~ms}$; Picker International, VISTR-MR 0.5 Tesla superconducting magnet showed abnormal signal intensity (SI) of the entire corpus callosum except for the extreme anterior portion. Very low SI was seen to extend from the genu to slightly posterior of the centre of the trunk of the corpus callosum, around which there was a border region with SI somewhat lower than that of the normal corpus callosum. Varying degrees of low SI were seen throughout the trunk and splenium (fig 1).

Positron emission tomography (PET) was performed three and four months after onset. The cerebral metabolic rate for oxygen $\left(\mathrm{CMRO}_{2}\right)$ and cerebral blood flow (rCBF) were measured using the ${ }^{15} \mathrm{O}$-steady state method. No relevant differences between the cerebral hemispheres were found.

\section{CROSSED HOMONYMOUS HEMIANOPIA}

Two months after onset, a left homonymous hemianopia was noted both on confrontation tests (verbal response) and on the Goldmann perimeter with the right hand pushing the switch (fig 2A). Four months after onset, she was assessed using the Goldmann perimeter with the left hand pushing the switch. A definite right homonymous hemianopia was found; that is, both hands showed a "crossed homonymous hemianopia" (fig 2B). From eight months after onset, the Goldmann perimeter test and the OCTOPUS static automated perimeter test indicated a right homonymous hemianopia with either hand (fig 2C). Confrontation tests conducted at the same time consistently indicated a left homonymous hemianopia so that the initial crossed homonymous hemianopia with the left and right hands was found to be tran-

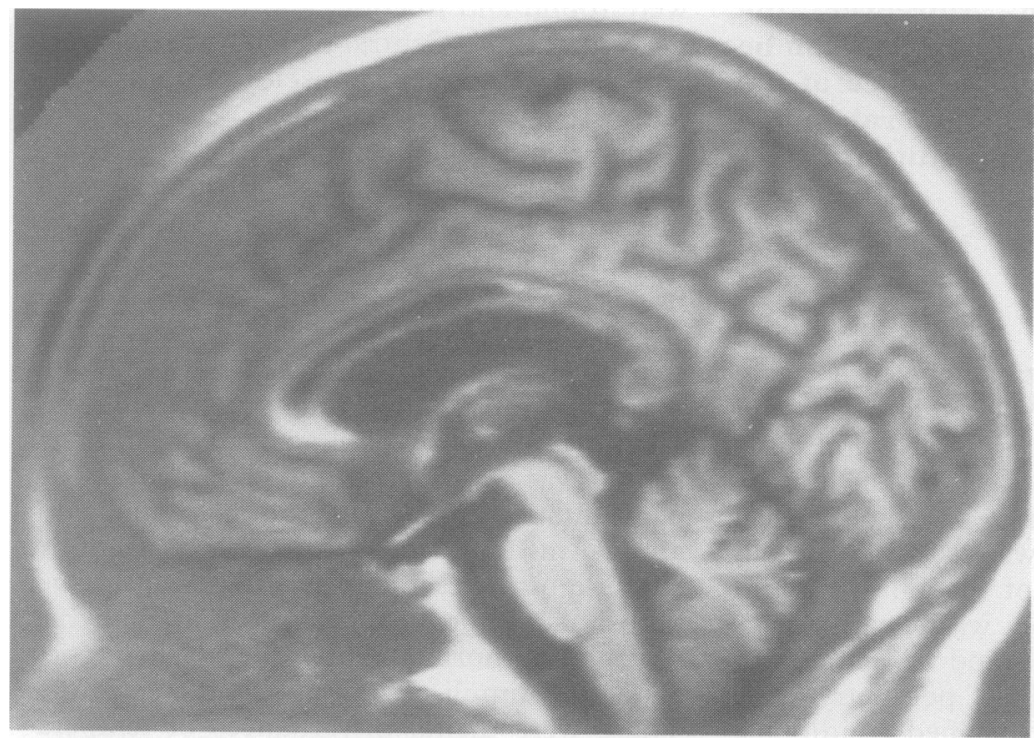

Figure 1 T1-weighted sagittal MRI (Repetition time $2080 \mathrm{~ms}$. Inversion time $500 \mathrm{ms,}$ inversion recovery technique) on the 34th day. The most anterior portion of the corpus callosum shows normal SI, whereas the SI from the genu to the anterior half of the trunk shows a long and thin very low SI, around which there is a border of tissue with SI somewhat lower than that of normal callosal tissue. In other regions, the corpus callosum shows heterogeneous low SI. sient. Later, results of confrontation tests and those obtained by using more sophisticated instruments, such as the Goldmann perimeter test, continued to be contradictory.

At seven months, when instructed to imitate a simple finger pattern presented with one hand ipsilaterally, no deficit (34/36 with the right hand, $36 / 36$ with the left hand) was found. She rarely failed to imitate simple finger patterns presented in the left visual fieldwhere the examiner's hand should not have been visible in confrontation tests. When required to imitate a contralateral finger pattern, she failed to imitate any pattern with either hand.

The response to placing a rod in the left visual field was also tested. When asked if she could see the rod, she said she could not, but when asked to grasp it, she was able to do so swiftly and accurately with the left hand. She wondered why she could grasp it, despite the fact that she couldn't see it. When asked to grasp the rod in the left visual field with the right hand, she would repeatedly grope in mid-air-eventually sometimes hitting it. In contrast, when the rod was placed in the right visual field, she said she could see it and had no difficulty in grasping it with the right hand, but clearly had difficulties with the left hand.

Four years after onset, there is no change in the pointing pattern and she is still unable to grasp a rod placed in the contralateral visual field. However, she says she can now see objects in the left visual field and is able to indicate the number of fingers displayed to her in that field.

\section{CROSSED LEFT HEMISPATIAL NEGLECT}

In Albert's line-crossing test, ${ }^{1}$ there was marked left-sided visual neglect with the right hand, but no indication of visual neglect with the left hand (fig 3). It was evident that there was neglect of the left hand side when copying figures with the right hand. Fig $4 \mathrm{~A}$ shows the patient's drawings of a Greek cross. She drew a winding cross with the left hand, but a straight one omitting the left part with the right hand. Fig 4B shows her drawings of two flowers in a pot. With the right hand, the flower on the left and the petals on the left of the other flower were omitted, indicating left visual neglect. However, although drawing with the left hand was somewhat disordered, neglect was not evident. Fig 4C shows her drawings of a house and two trees on both sides of it. She failed to draw the left part of the house. When asked to read a Chinese character (kanji) out aloud, she read out only the right half of the character and neglected the left half which in many cases modifies the right half, changing its meaning. The above findings were observed not only when the original was placed directly in front of her, but also when it was placed on her right or left side and she was instructed to draw with her right or left hand. The results were not considerably different from those found when the paper was placed directly in front of her. In drawing a woman's face without a 
A

2nd month

B

C

8th month

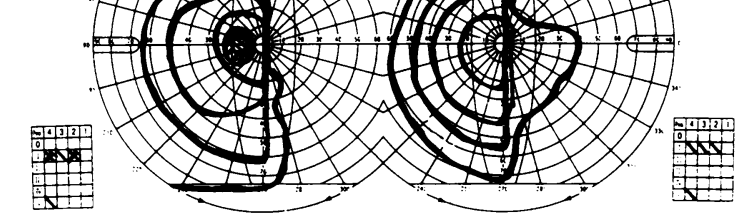

Left hand
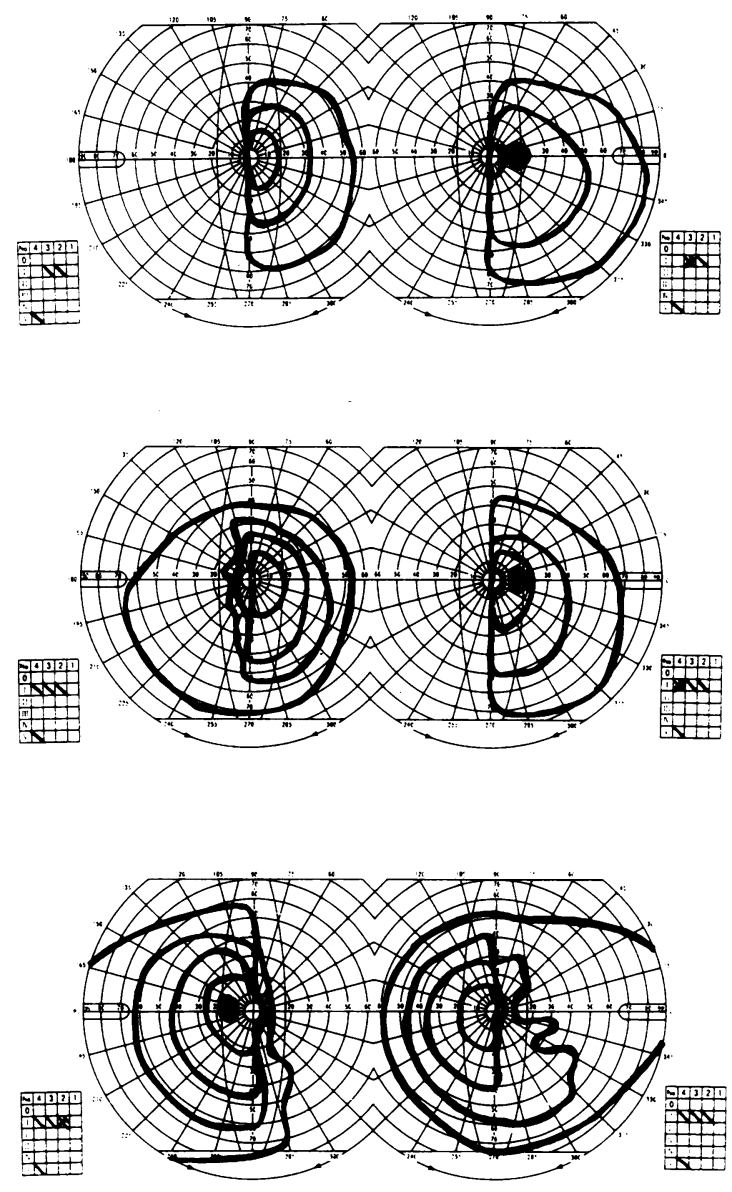

Right hand

Figure 2 Goldmann perimeter tests (2, 4 and 8 months after onset). Results on the left (right) hand side of the figure are those obtained with the left (right) hand pressing the switch. After two months, a left homonymous hemianopia is found with the right hand pressing the switch $(A)$, whereas after four months a left homonymous hemianopia is found with the right hand pressing the switch while a right homonymous hemianopia is found when the left hand did so, that is "crossed homonymous hemianopia" (B). After eight months, a pattern similar to that after 4 months is seen with the left hand, but, with the right hand, the visual field defect has become a right homonymous hemianopia (C).

Figure 3 Albert's linecrossing test. With the left hand, no hemispatial neglect is found. But, with the right hand, dramatic hemispatial neglect is found. model, unilateral neglect was not seen with either hand (fig 5). Slight "crossed left hemispatial neglect" still remains four years from onset.

Other signs of the interhemispheric disconnection syndrome, that is, apraxia and tactile anomia of the left hand, were also observed. Agraphia of the left hand was found when attempting to write Chinese characters and Japanese script. The nature of the mistakes made were not due to scrawling with the nondominant hand, but mainly due to paragraphia with perseveration. In a dichotic listening test there was clear extinction of the

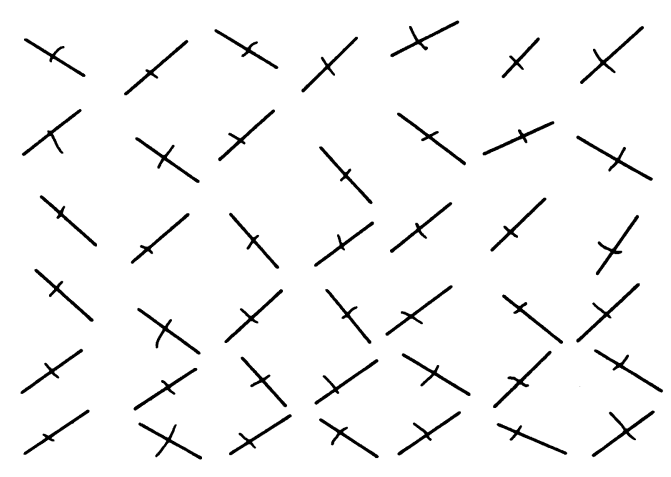

Left hand left ear. Facial recognition, identification of overlapping figures (Poppelreuter), colour naming, left-right discrimination, finger naming and simple verbal calculation were intact. The Wechsler Adult Intelligence Scale (WAIS) three months after onset, showed a verbal IQ of 73, a performance IQ below 60 and total score below 60 . In the Western Aphasia Battery ${ }^{2}$ one year after onset, no significant abnormalities were seen concerning her ability to write except for her occasional refusal to do so. Construction tasks with the right hand were moderately impaired and those with the left hand were slightly better.

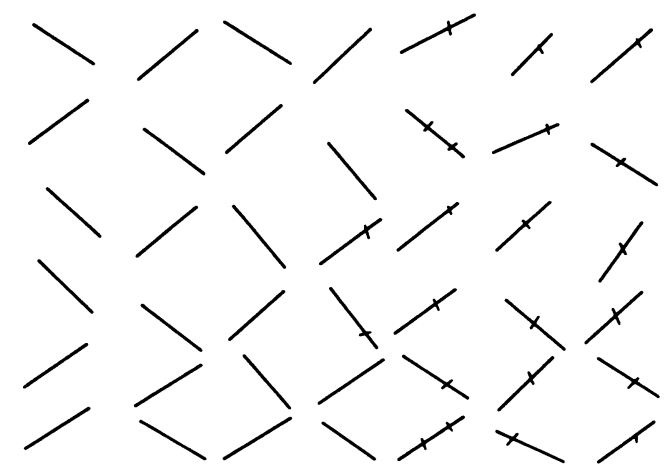

Right hand 
Figure 4 Copying models with the left and right hands. A: Greek cross. With the left hand, the cross is curved. With the right hand, the left part is omitted. B: Two flowers in a pot. The drawing with the left hand is less precise, but there is no neglect, whereas with the right hand, the figure is copied showing left hemi-neglect (complete omission of the flower on the left and also the left-half of the flower on the right). C: $A$ house with 2 trees on both sides of it. With the right hand, the left part of the house is not drawn.
Figure 5 Drawings of a woman's face without a model. Even with the right hand, hemispatial neglect is not clear.
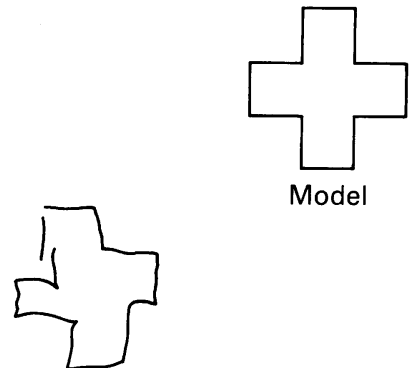

A

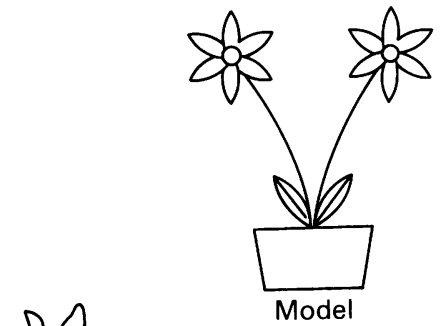

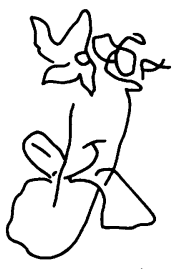

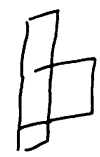

Right hand

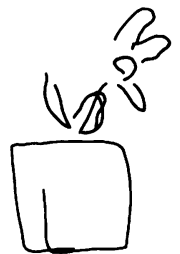

Right hand

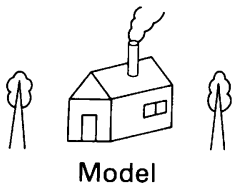



C

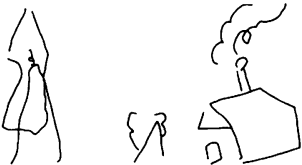

Right hand
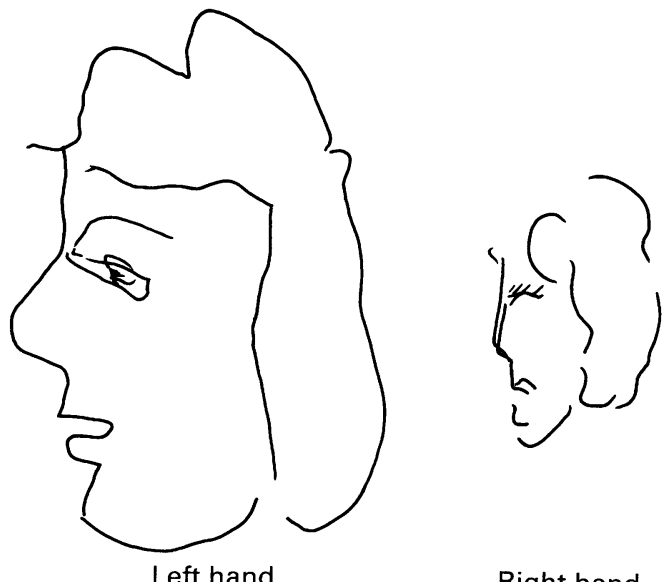

Right hand
No other abnormalities were found and there was no aphasia.

\section{Discussion}

There was no difficulty in diagnosing Marchiafava-Bignami disease from the history of alcohol abuse, the pathognomonic findings on $X$ ray CT and MRI and the various signs of the interhemispheric disconnection syndrome, such as apraxia, agraphia and tactile anomia of the left hand.

The most characteristic feature of this case is the crossed homonymous hemianopia. After four months, a left homonymous hemianopia was found using the Goldmann perimeter with the right hand pushing the switch, but a right homonymous hemianopia was found with the left hand. This "crossed homonymous hemianopia" can be explained by a failure of unilaterally-presented visual information travelling across the corpus callosum to the contralateral motor system, to initiate a response. After eight months, confrontation tests indicated a left homonymous hemianopia, whereas the tests using instruments such as the Goldmann kinetic perimeter or OCTOPUS static perimeter with either hand indicated a right homonymous hemianopia. These results may have been due to different levels of hemispheric activation by the two kinds of tests. That is, in tests requiring verbal responses, the left hemisphere may be more highly activated, whereas in tests requiring only the tracking of a simple light source, the right hemisphere may be more highly activated. This might then explain why two distinct forms of crossed homonymous hemianopia were observed. When the crossed homonymous hemianopia was initially found, there may have been a complete lack of interhemispheric communication and the results would have reflected the functions of the isolated left and right hemispheres. The crossed homonymous hemianopia observed later would then have been a result of the different hemispheric activation induced by the different testing methods. After four years, the left homonymous hemianopia observed on confrontation and the right homonymous hemianopia observed with Goldmann perimeter testing are both less distinct, suggesting that there has been some recovery of interhemispheric communication, and that the crossed homonymous hemianopia will continue to improve. Despite the fact that for three years the patient said she "could not see" objects in the left visual field, she could readily and accurately grasp them with the left hand, and she was able to imitate finger patterns presented by the examiner to the left visual field with the left hand. From these results, we believe that this patient is neither able to verbalise objects seen in the left visual field nor is she conscious of them, in spite of the fact that there is essentially no disturbance of her visual field.

We have been unable to find any previous reports of crossed homonymous hemianopia 
detected by visual field tests using the Goldmann perimeter or comparable instruments, but several related syndromes have been reported. Brion and Jedynak ${ }^{3}$ reported a case of a callosal vascular lesion, due to a left paracallosal angioma fed by the posterior cerebral artery, showing a so-called "pseudohémianopsie". Notably, they found that when two objects were placed one each in the left and right visual fields, the object on the left was ignored. The presence of the stimulus on the left was sometimes denied, but their patient could readily and, simultaneously, grasp the objects with the left and right hands respectively, indicating that the object in the left visual field was seen. Our patient consistently denied that she could see the object in the left visual field, but was easily able to grasp it. This phenomenon was not due to extinction. Lhermitte et $a l^{4}$ reported three cases of suspected MBD, in which verbal responses could not be elicited following the presentation of a stimulus in the left visual field or placement of an object in the left hand, but when instructed to raise the left hand to indicate a response, the instruction was obeyed. They called this syndrome "pseudo-extinction", because it differed from the case reported by Brion and Jedynak ${ }^{3}$ which involved two stimuli in separate fields. However, Lhermitte's case and our case are similar as correct responses could be elicited by non-verbal means. Neither of these reports, however, mentions crossed visual field deficits. In a study of a case of $\mathrm{MBD}$ at necropsy, Lechevalier et al ${ }^{5}$ reported a "crossed avoiding reaction", in which a hand would actively avoid an object placed in the contralateral field. Their case was, however, different from our case as no abnormalities on the Goldmann perimeter test could be found. Bogen ${ }^{6}$ noted "double hemianopia" as one of the signs of the IDS. He described this syndrome as the ability of the subject sitting opposite the examiner to point to the examiner's finger when it was placed individually in the visual field of the subject's pointing hand, but the inability to point if the examiner's finger was placed in the visual field contralateral to the subject's pointing hand; at which time the subject would completely ignore it, as in homonymous hemianopia. Moreover, when the examiner used both hands in both visual fields, the subject was able to point simultaneously to both fingers. Similar results were obtained in this case using the same examination technique, but such results leave unclear what differences there may be with callosal crossed visual ataxia. Bogen ${ }^{6}$ referred to this syndrome as being similar to homonymous hemianopia, but he reported neither the results of confrontation tests nor the results of Goldmann perimeter tests. Irrespective of the fact that she could not see objects in the left hemifield, her ability to grasp each object accurately resembles cases of blindsight including the DB case described by Weiskrantz. ${ }^{7}$

One of the most notable characteristics of our case is that, despite an absence of neglect when copying simple pictures, figures or Chinese characters with the left hand, there is a notable left hemispatial neglect with the right hand. Even in Albert's line-crossing test ${ }^{1}$ with either hand, only the right hand showed clear left hemispatial neglect. When reading Chinese characters, she omitted the left part entirely and read only the right half of those characters whose right halves could be read as independent entities. Neither hand showed abnormalities during spontaneous drawing. These findings suggest that the deficit is in the process of visual input. This "crossed left hemispatial neglect" is not mentioned in neuropsychology texts $^{89}$ or in reviews of interhemispheric disconnection syndrome. ${ }^{610}$ However, similar signs were reported by Sine et al, ${ }^{11}$ in a case of left parasagittal frontoparietal haemorrhage with spasm of the anterior cerebral artery and by Goldenberg et al ${ }^{1213}$ in a case of pericallosal haemorrhage of the anterior two thirds of the corpus callosum, both cingulate gyri and the white matter underlying both medial frontal lobes secondary to bleeding from an aneurysm of the anterior cerebral artery. Recently, Kashiwagi et $a l^{14}$ noted a similar case secondary to infarction of the anterior cerebral artery. Furthermore, Costello and Warrington ${ }^{15}$ reported both a right-sided visuospatial neglect and left-sided neglect dyslexia (misreading the beginning of a word) in a case of lymphoma occupying mainly the left occipital lobe including the splenium.

"Crossed left hemispatial neglect" can be explained as follows: in our case, neither hemispace is neglected in copying with the left hand, although the right hemisphere alone is functioning; in copying with the right hand, however, attention is paid exclusively to the contralateral right hemispace and the ipsilateral left hemispace is neglected because only the left hemisphere which is disconnected from the right, is functioning. "Crossed left hemispatial neglect" has not previously drawn attention as one of the signs of the interhemispheric disconnection syndrome.

In our case, PET was performed. No significant lateralisation, however, was found in terms of either rCBF or oxygen metabolic rate. PET and MRI results also indicate that the "crossed homonymous hemianopia" and "crossed left hemispatial neglect" observed are not due to lesions of the cerebral hemispheres.

1 Albert ML. A simple test of visual neglect. Neurology 1973;23:658-64.

2 Kertesz A. The Western Aphasia Battery. New York: Grune and Stratton, 1982.

3 Brion S, Jedynak CP. Troubles du transfert interhémisphérique (callosal disconnection). A propos de trois pherique (callosal disconnection). A propos de trois main étrangère. Rev Neurol (Paris) 1972;126:257-66.

4 Uhermitte F. Marteau R, Serdaru M, Chedru F. Signs of interhemispheric disconnection in Marchiafava-Bignami disease. Arch Neurol 1977;34:254.

5 Lechevalier B, Andersson JC, Morin P. Hemispheric disconnection syndrome with a 'crossed avoiding' reaction in a case of Marchiafava-Bignami disease. $\mathcal{f}$ Neurol Neurosurg Psychiatry 1977;40:483-97.

6 Bogen JE. The callosal syndrome. In: Heilman KM, Valenstein E, eds. Clinical neuropsychology. Oxford: Oxford University Press 1979:308-59.

7 Weiskrantz L. Blindsight - a case study and implications. 
Oxford: Oxford University Press 1990:20-46.

8 Hécaen $\mathrm{H}$, Albert ML. Disorders of visual perception. In: Hécaen $\mathrm{H}$, Albert $\mathrm{ML}$, eds. Human Neuropsychology. New York: John Wiley, 1978:217-24.

9 Heilman KM, Valenstein E, Watson RT. The neglect syndrome. In: Frederiks JAM, eds Handbook of clinical neuropsychology. Amsterdam: Elsevier Science 1985:153-83.

10 Sperry RW, Gazzaniga MS, Bogen JE. Interhemispheric relationships: the neocortical commissures; syndromes relationships: the neocortical commissures; syndromes of hemisphere disconnection. In: Vinken PJ, Bruyn Amsterdam: North-Holland 1969:273-90.

11 Sine RD, Soufi A, Sham M. Callosal syndrome:
Implications for understanding the neuropsychology of stroke. Arch Phys Med Rehabil 1984;65:606-10.

12 Goldenberg G, Wimmer A, Holzner F, Wessely P. Apraxia of the left limbs in a case of callosal disconnection: The contribution of medial frontal lobe damage. Cortex 1985;21:135-48.

13 Goldenberg G. Neglect in a patient with partial callosal disconnection. Neuropsychologia 1986;24:397-403.

14 Kashiwagi A, Kashiwagi T, Nishikawa T, Tanabe $H$ Okuda J. Hemispatial neglect in a patient with callosal Okuda J. Hemispatial neglect in a pat

15 Costello A, Warrington EK. The dissociation of visuospatial neglect dyslexia. $尹$ Neurol Neurosurg Psychiatry 1987;50:1110-6. 